Respiratory Society/World Association of Sarcoidosis and other Granulomatous Disorders. Sarcoidosis Vasc Diffuse Lung Dis 1999; 16: 149-173.

5 Huez S, Retailleau K, Unger P, et al. Right and left ventricular adaptation to hypoxia: a tissue Doppler imaging study. Am J Physiol Heart Circ Physiol 2005; 289: H1391-H1398.

6 Garcia MJ, Rodriguez L, Ares M, et al. Myocardial wall velocity assessment by pulsed Doppler tissue imaging: characteristic findings in normal subjects. Am Heart J 1996; 132: 648-656.

7 Lindqvist $\mathrm{P}$, Waldenström A, Wikström G, et al. The use of isovolumic contraction velocity to determine right ventricular state of contractility and filling pressures: A pulsed Doppler tissue imaging study. Eur J Echocardiogr 2005; 6: 264-270.
8 Nunes H, Humbert M, Capron F, et al. Pulmonary hypertension associated with sarcoidosis: mechanisms, haemodynamics and prognosis. Thorax 2006; 61: 68-74.

9 Lamberto C, Nunes H, Le Toumelin P, et al. Membrane and capillary blood components of diffusion capacity of the lung for carbon monoxide in pulmonary sarcoidosis: relation to exercise gas exchange. Chest 2004; 125: 2061-2068.

10 Hunter KS, Lee PF, Lanning CJ, et al. Pulmonary vascular input impedance is a combined measure of pulmonary vascular resistance and stiffness and predicts clinical outcomes better than pulmonary vascular resistance alone in pediatric patients with pulmonary hypertension. Am Heart J 2008; 155: 166-174.

DOI: 10.1183/09031936.00065210

\title{
Interferon- $\gamma$ release assays in tuberculosis contacts: is there a window period?
}

The tuberculin skin test (TST) is the established procedure for diagnosis of latent tuberculosis infection (LTBI) among the contacts of an infectious tuberculosis (TB) case. TST may convert to positive $\leqslant 8$ weeks after Mycobacterium tuberculosis infection, an interval that is usually referred to as the "window period". A negative TST obtained $<8$ weeks before does not exclude infection, and a second test is recommended [1, 2]. However, TST has some limitations, such as cross-reactivity with Bacille Calmette-Guérin (BCG) and with nontubercular mycobacterial infections.

T-cell interferon- $\gamma$ release assays (IGRA) are emerging as new screening tools for LTBI diagnosis. IGRAs incorporate specific antigens of $M$. tuberculosis that are absent in BCG strains and in the majority of nontubercular mycobacteria, offering enhanced specificity for detecting $M$. tuberculosis infection [3]. In addition, their use has been approved for screening of infection in contacts $[1,4]$. Most TB contact studies have shown a better correlation of IGRA with the intensity of $M$. tuberculosis exposure than that obtained using TST, particularly in patients previously vaccinated with BCG [5]. Yet, to our knowledge, no study has considered carefully the window period after $M$. tuberculosis exposure while simultaneously evaluating responses for TST and IGRA. The aim of this study was to find out which of the two tests converts earlier to positive in persons with recent infection after contact with an infectious TB case.

We used a longitudinal prospective analysis to study 184 healthy adults, all having had recent contact with a microbiologically confirmed pulmonary $\mathrm{TB}$ index patient. The setting was a specialized TB clinic in Pontevedra, Spain, where the incidence of TB has historically been among the highest in Western Europe. In 1996, a TB control programme was established, and incidence has steadily dropped from 72.3 to 32.6 per 100,000 in 2008 .

TST and a commercially available IGRA, Quantiferon $($ ) TB Gold In-Tube (QFT; Cellestis, Carnegie, Australia), were both carried out at the first visit of the contacts to our clinic after TB exposure (time 0: TST0 and QFT0, respectively). The attending physician was blind to the QFT result, and medical advice was offered to the patient only on the basis of the TST result. Patients with positive TST were offered to start LTBI chemotherapy after active TB was excluded. For those contacts with a negative TST0, a repeat TST and QFT were undertaken 2 months later (time 2: TST2 and QFT2, respectively), at the end of the window period. In addition, patients with a positive TST0 but negative QFT0 were recalled to have a second QFT 2 months later, irrespective of whether the patient was receiving treatment for LTBI.

Peripheral blood was processed for the QFT assay according to the manufacturer's instructions. Samples were frozen and stored at $-70^{\circ} \mathrm{C}$ for analysis $3-4$ weeks later. The cut-off value for a positive test was $0.35 \mathrm{IU} \cdot \mathrm{mL}^{-1}$. TST was carried out according to the Mantoux method, with 2 units of tuberculin RT-23 (purified protein derivative; State Serum Institute, Copenhagen, Denmark), following the standardised protocol. A positive TST was defined as an induration of $\geqslant 5 \mathrm{~mm}$. We excluded from the study HIV-infected persons or those with other immunosuppressive conditions, pregnant females, and those with a previous documented positive TST. Institutional ethical approval was obtained from the Ethical Committee of Clinical Research (Xunta de Galicia, Spain). All participants included in the study gave their written informed consent.

Initially, 184 participants, recent contacts of microbiologically confirmed pulmonary TB patients were enrolled in the study, but 32 were excluded due to a history of a previous positive TST (28 cases) or because of incomplete data for the index TB case (four cases). The remaining 152 participants had a median age of 44 yrs (interquartile range 32-55 yrs) and were contacts of 48 different index TB patients. None had ever previously received TB or LTBI treatment. $55(36.2 \%)$ contacts had had a previous BCG vaccination. For 137 (90.1\%) contacts, the index TB case was acid-fast bacilli positive. 
The results of our study are summarised in figures 1 and 2 . At the first visit, $62(40.8 \%)$ contacts showed a positive TST0, while $57(37.5 \%)$ contacts had a positive QFT0. A total of 90 contacts with an initial negative TST0 $(0 \mathrm{~mm})$ were called 2 months later for TST2 and QFT2 studies, which were finally performed on 81 patients (nine patients did not return after 2 months). Of those contacts, 24 converted to a positive TST2, representing $29.6 \%(95 \%$ CI $19.1-40.2 \%)$. It is worth noting that nine of them (37.5\%, 95\% CI 16.0-59.0\%) had already been positive in QFT0, suggesting an earlier conversion of QFT than TST.

In 80 participants with an initial negative QFT0 (12 with positive TST0 and 68 with negative TST0), a second QFT2 was also carried out. Of this group, 17 converted to positive $(21.2 \%$, 95\% CI 11.7-30.8\%), including three who had had a positive TST0 $(17.6 \%$, 95\% CI $3.8-43.4 \%)$, while a further 12 showed

a)

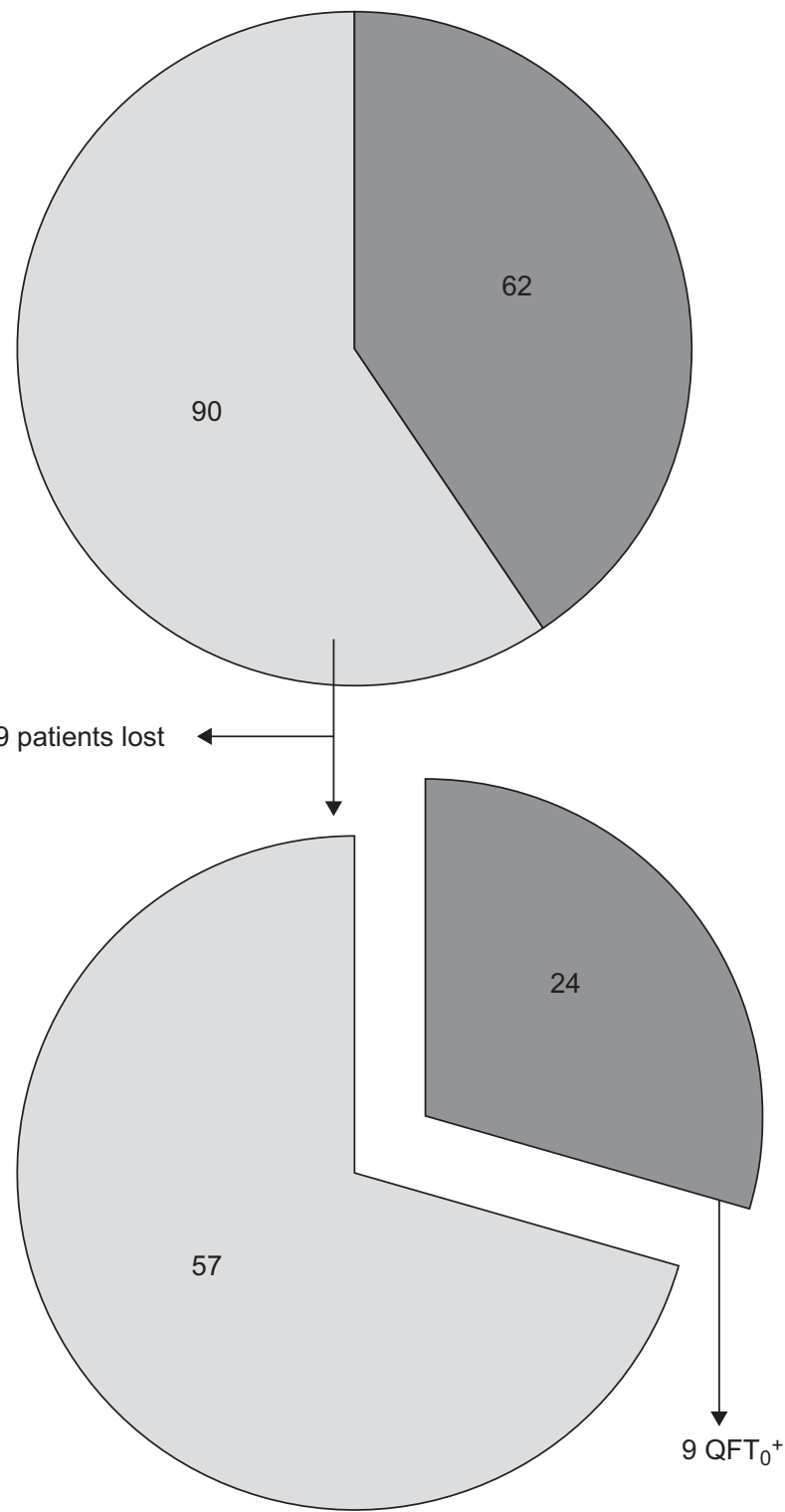

both TST and QFT conversion. In two cases, negative at TST0 and TST2, both had QFT2 results slightly above the diagnostic cut-off limit suggested by the manufacturer $(0.37$ and $0.45 \mathrm{IU} \cdot \mathrm{mL}^{-1}$, respectively).

Our results indicate that, as for TST, QFT has a window period of conversion after exposure to $M$. tuberculosis. Time of conversion may differ between the two, but still has to be clearly determined for QFT. Nevertheless, QFT conversion preceded that of TST in $37.5 \%$ of participants with documented TST conversion, while TST was found to be positive at the first visit after exposure in only three out of 17 cases with QFT conversion, suggesting that an earlier TST conversion is also possible. In at least two of these cases, we cannot rule out that a positive TST0 could be related to previous TB infections or BCG vaccination. b)

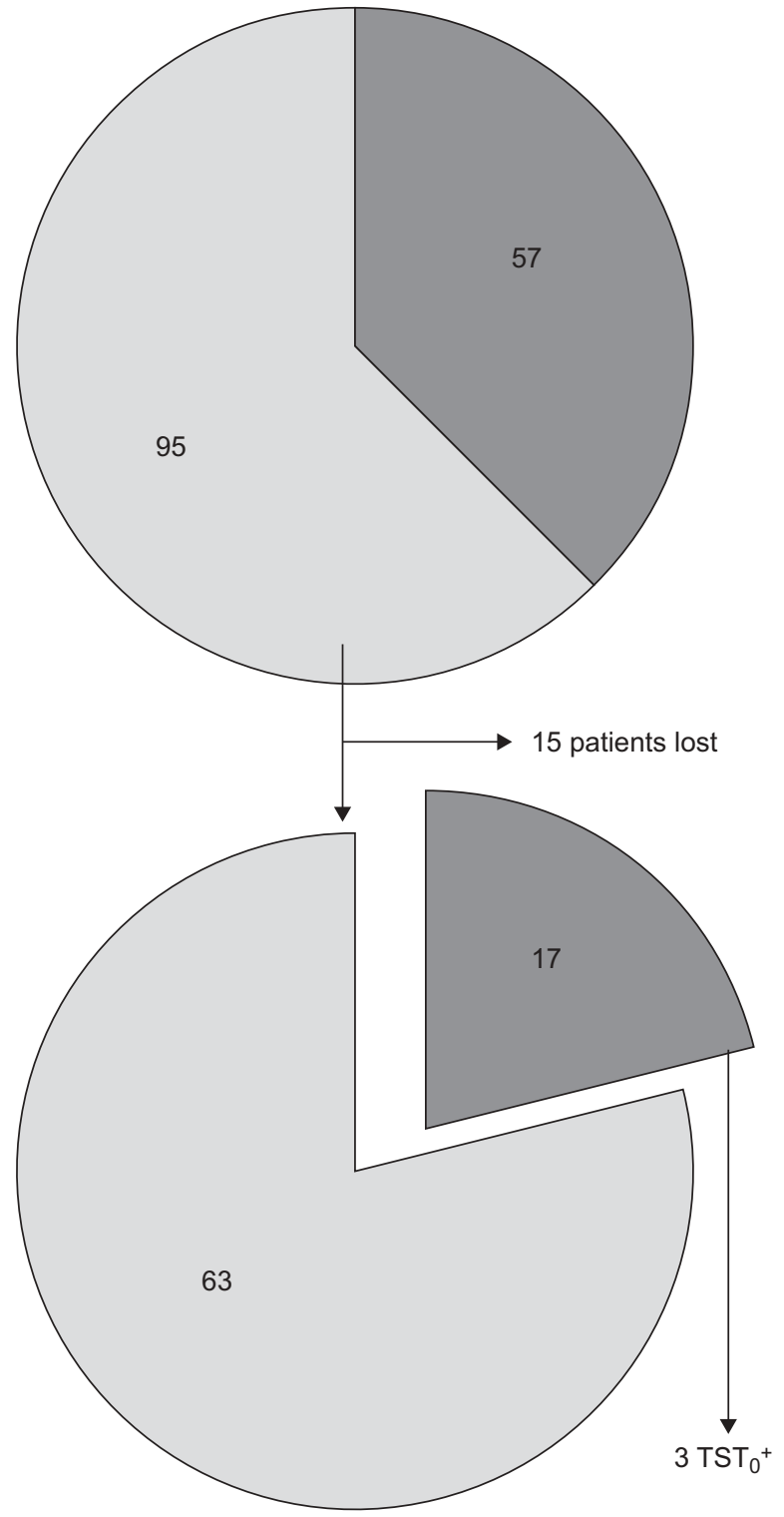

FIGURE 1. Results of $a, c)$ tuberculin skin test (TST) and b, d) Quantiferon $\mathfrak{B}_{\mathbb{B}}$-TB Gold In-Tube (QFT) assay after a, b) recent contact with a tuberculosis index case (TSTo and QFTo) and c, d) after the 2-month window period (TST2 and QFT2). a) $n=152 . b) n=152 . c) n=81 . d) n=80$. Data are presented as contacts $n$. 1 : : negative test result; 口: positive test result. 


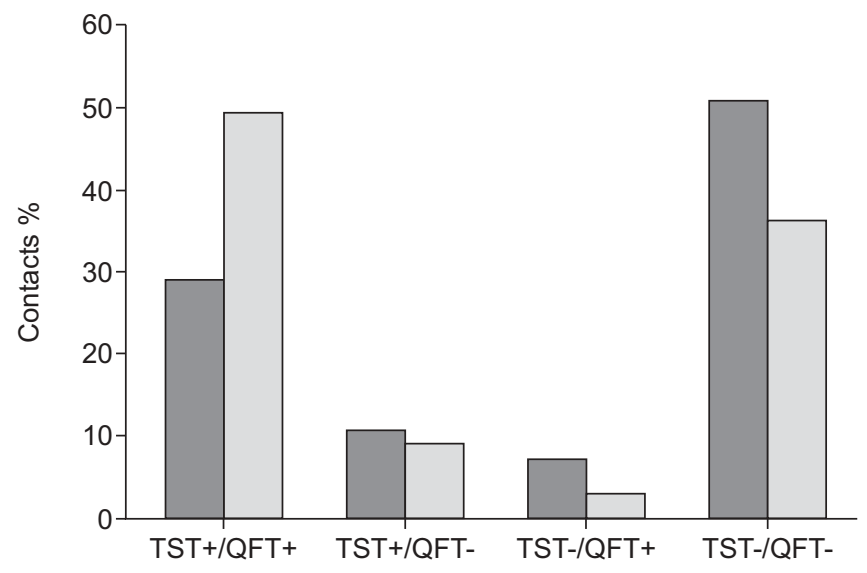

FIGURE 2. Results of tuberculin skin test (TST) and Quantiferon $\mathbb{B}_{\mathbb{B}}$-TB Gold InTube assay (QFT) after recent contact with a tuberculosis index case $(\square)$ and 8 weeks later $(\square)$.

Our findings suggest that IGRAs could be a valid alternative to TST in the study of TB contacts, especially with increasing evidence that a positive IGRA result is a predictive factor for progression to TB [6, 7]. Nevertheless, to the best of our knowledge, this is the first study that simultaneously compares TST and an IGRA, looking for a possible conversion in both tests. Although the time of QFT conversion still has to be clearly determined, our work contributes towards clarifying the time for studying contacts with IGRAs.

Our study has some limitations that deserve comment. First, there is some concern about the possibility that a prior skin test could boost the response of subsequent IGRAs [8]. In theory, this could invalidate some of the positive IGRA responses obtained 2 months after the TST, but other authors have reported no effect [9]. A second limitation is that QFT reversion to negative in patients with an initial positive QFT0 cannot be ruled out. In addition to the spontaneous reversion reported for TST responses, IGRA reversion has also been described in TB contacts, and its significance is still a matter of debate [10].

From the results of our study, we conclude that QFT has a "window period" after exposure to M. tuberculosis. Therefore, for those cases with an initially negative result with QFT after recent exposure, a later second test must be carried out to definitely exclude infection. Alternatively, when QFT is the only technique used, the last possible exposure time to the index case can be assessed, and blood is only drawn for the QFT after an 8-week interval.

\section{Anibarro*, M. Trigo", C. Villaverde", A. Pena*, S. Cortizo", D. Sande*, R.A. Pazos* and Á. González-Fernández ${ }^{+}$}

*Tuberculosis Unit, Dept of Infectious Diseases and Internal Medicine, "Dept of Microbiology, Pontevedra Hospital Complex, Pontevedra, "Dept of Statistics and Operational Research, and ${ }^{+}$Immunology and Joint Immunology Unit, Vigo University Teaching Hospital, University of Vigo, Vigo, Spain.

Correspondence: L. Anibarro, Unidade de Tuberculose, Complexo Hospitalario de Pontevedra, Hospital Provincial, Rúa Loureiro Crespo no. 2. 36001-Pontevedra, Spain. E-mail: luis.anibarro.garcia@sergas.es

Support Statement: The study received financial support from the SUDOE-FEDER (IMMUNONET-SOE1/1P1/E014) and Spanish Ministry of Science and Innovation (Consolider Ingenio 2010, CSD2006-12, NANOBIOMED).

Statement of Interest: None declared.

\section{REFERENCES}

1 National Tuberculosis Controllers Association, Centers for Disease Control and Prevention, Guidelines for the investigation of contacts of persons with infectious tuberculosis. Recommendations from the National Tuberculosis Controllers Association and CDC. MMWR Recomm Rep 2005; 54: 1-47.

2 Menzies D. Interpretation of repeated tuberculin tests. Boosting, conversion, and reversion. Am J Respir Crit Care Med 1999; 159: 15-21.

3 Pai M, Zwerling A, Menzies D. Systematic review: T-cell-based assays for the diagnosis of latent tuberculosis infection: an update. Ann Intern Med 2008; 149: 177-184.

4 Ruiz-Manzano J, Blanquer R, Calpe J, et al. [Diagnosis and treatment of tuberculosis]. Arch Bronconeumol 2008; 44: 551-566.

5 Arend S, Thijsen S, Leyten E, et al. Comparison of two interferon- $\gamma$ assays and tuberculin skin test for tracing tuberculosis contacts. Am J Respir Crit Care Med 2007; 175: 618-627.

6 Diel R, Loddenkemper R, Meywald-Walter K, et al. Predictive value of a whole blood IFN- $\gamma$ assay for the development of active tuberculosis disease after recent infection with Mycobacterium tuberculosis. Am J Respir Crit Care Med 2008; 177: 1164-1170.

7 Kik S, Franken W, Mensen M, et al. Predictive value for progression to tuberculosis by IGRA and TST in immigrant contacts. Eur Respir J 2010; 35: 1346-1353.

8 van Zyl-Smit R, Zwerling A, Dheda K, et al. Within-subject variability of interferon- $\gamma$ assay results for tuberculosis and boosting effect of tuberculin skin testing: a systematic review. PLoS One 2009; 4: e8517.

9 Leyten E, Prins C, Bossink A, et al. Effect of tuberculin skin testing on a Mycobacterium tuberculosis-specific interferon- $\gamma$ assay. Eur Respir J 2007; 29: 1212-1216.

10 Pai M, Joshi R, Dogra S, et al. T-cell assay conversions and reversions among household contacts of tuberculosis patients in rural India. Int J Tuberc Lung Dis 2009; 13: 84-92.

DOI: $10.1183 / 09031936.00030610$ 